Pacific Journal of Mathematics

ON THE HIGMAN-SIMS SIMPLE GROUP OF ORDER 


\title{
ON THE HIGMAN-SIMS SIMPLE GROUP OF ORDER 44,352,000
}

\author{
DAVID ParrotT AND S. K. WONG
}

In a recent paper D. G. Higman and C. C. Sims announced their construction of a new simple group $H_{100}$ of order 44,352,000. The group $H_{100}$ is obtained as a rank 3 permutation group of degree 100 with subdegrees 1,22 and 77 ; and the stabilizer of a point is isomorphic to the Mathieu simple group $M_{22}$. Shortly after their announcement of the new simple group, Graham Higman constructed a simple group of the same order as a doubly transitive group of degree 176 and with stabilizer of a point isomorphic to $P S U\left(3,5^{2}\right)$.

The purpose of this paper is to show that the two groups mentioned above are isomorphic, and in fact, that there is exactly one (up to isomorphism) simple group of order 44,352,000.

THEOREM. Let $G$ be a nonabelian simple group of order 44,352,000. Then $G$ is isomorphic to the Higman-Sims group $H_{100}$.

Throughout this paper, $G$ will denote a nonabelian simple group of order $44,352,000=2^{9} \cdot 3^{2} \cdot 5^{3} \cdot 7 \cdot 11$. The notation will be standard; see for instance [13]. Further, a Sylow $p$-subgroup of $G$ will be denoted by $G_{p}, A_{n}$ and $S_{n}$ will denote the alternating group and symmetric group on $n$ letters respectively; and $F_{20}$ will denote a Frobenius group of order 20. The word "character" always refers to an irreducible character of $G$ afforded by an irreducible representation of $G$ in the complex number field. If the integer $n$ divides the $|G|$ of $G$, we will denote this by $n|| G \mid$.

In the proof of the theorem, the following results are of fundamental importance.

Result 1 (R. Brauer [2], Theorem 11). Let $G$ be a group such that $p|| G \mid$, but $p^{2} \nmid|G|, p$ a prime. If the $p$-block $B_{1}(p)$ contains the principal character $1_{G}$ of $G$, then $B_{1}(p)$ has $(p-1) / t$ (irreducible complex) characters which are $p$-conjugate only to themselves and one family of $t p$-conjugate characters, where $t$ denotes the number of conjugate classes of elements of order $p$. Further, the degrees $\chi_{i}(1)$ of the irreducible characters $\chi_{i}$ of $B_{1}(p)$, satisfy the following congruences:

$$
\chi_{i}(1)=z_{i} \equiv \delta_{i}= \pm 1(\bmod p),
$$

if $\chi_{i}$ is $p$-conjugate only to itself and

$$
\chi_{i}(1)=z_{i} \equiv \frac{\delta_{i}}{t}= \pm\left(\frac{1}{t}\right)(\bmod p),
$$


if $\chi_{i}$ belongs to the family of $p$-conjugate characters If $1_{G}=\chi_{1}, \chi_{2}, \cdots$, $\chi_{q+1}$ represent the different families of $B_{1}(p),(q=(p-1) / t)$, then

$$
1+\delta_{2} z_{2}+\cdots+\delta_{q+1} z_{q+1}=0 .
$$

The next result is very well-known. (For a proof, see [8] (2.15), or any book on group theory).

RESULT 2. Let $K_{1}, K_{2}, K_{3}$ be any three conjugate classes of elements of a finite group $G$. Let $x_{3} \in K_{3}$ and let $a\left(x_{1}, x_{2} ; x_{3}\right)$ be the number of ordered pairs $\left(x_{1}, x_{2}\right), x_{1} \in K_{1}$ and $x_{2} \in K_{2}$, such that $x_{1} \cdot x_{2}=x_{3}$. Then, if $\chi_{1}, \cdots, \chi_{t}$ are all the irreducible complex characters of $G$, we have

$$
a\left(x_{1}, x_{2} . x_{3}\right)\left|C_{G}\left(x_{1}\right)\right|\left|C_{G}\left(x_{2}\right)\right|=|G| \sum_{i=1}^{t} \frac{\chi_{i}\left(x_{1}\right) \chi_{i}\left(x_{2}\right) \overline{\chi_{i}\left(x_{3}\right)}}{\chi_{i}(1)} .
$$

Professor D. Wales has communicated the following result to the authors.

ResUlt 3. Let $G$ be a primitive permutation group of degree 100 with stabilizer of a point $H$ isomorphic to the Mathieu simple group $M_{22}$ and the orbits of $H$ are of length 1,22 and 77 . Then $G$ is isomorphic to the Higman-Sims simple group of order 44,352,000.

1. Determination of the Sylow $p$-normalizers for $p=11,7$ and 5. In this section, we will determine the Sylow 11-, 7- and 5- normalizers. Unfortunately the amount of numerical work required to show that the Sylow 11-subgroup is self-centralizing is too large to enable us to present the proof here. However, the methods and results used are similar to the examples of this kind of work given in [12] and [14]. In addition to the results given in [12] and [14], we also need some results of $R$. Brauer on the defect group of a block (see [3], [4] and [7], §86, 87, also [5], Theorem 2 and Theorem 3). Thus combining these methods we are able to show the following result.

Lemma 1.1. The Sylow 11-normalizer of $G$ is a Frobenius group of order 55.

Using equation (1), it is not difficult to show that the number of conjugate classes of elements of order 7 is one, i.e., $\left|N_{G}\left(G_{7}\right): C_{G}\left(G_{7}\right)\right|=6$. Then, with Lemma 1.1 and more numerical work, we have that $B_{1}(11) \cap B_{1}(7)=\{1,3200\}$, where the numbers in the brackets are the degrees of the irreducible characters which lie in both the principal 
11-block, $B_{1}(11)$ and the principal 7-block $B_{1}(7)$. If, in equation (2), we take $x_{1}=x_{2}=\nu$, where $\nu$ is an element of order 7 and $x_{3}$ is an element of order 11, then we have that $\left|C_{G}(\nu)\right| \mid 2.3 .7$. Hence by Sylow theorems, we have

LEMMA 1.2. The Sylow 7-normalizer of $G$ is a Frobenius group of order 42.

We now get the following possibilities for equation (1) for $B_{1}(11)$ and $B_{1}(7)$ :

Possibilities for equation (1) for $B_{1}(11)$ :

( I ) $1-3200-175+1750+2520-896^{*}=0$.

(II) $1-3200-175+1750+1750-126^{*}=0$.

(In case (I), the two 11-conjugate characters have the common degree 896 and in case (II), they have the common degree 126).

Possibilities for equation (1) for $B_{1}(7)$ :

(A) $1+3200-825-2750-1056+22+1408=0$.

(B) $1+3200-825-2750+22+22+330=0$.

(C) $1+3200-825-2750-55+330+99=0$.

(D) $1+3200-825-3520-1056+1408+792=0$.

Now let $\chi_{2}$ be the (unique) character of $G$ with degree 3200 . Then $\chi_{2}$ lies in a 5-block $B_{2}(5)$ say, of defect 1 . If $D$ is the defect group of $B_{2}(5)$, then $D$ has order 5 and $0_{5}\left(C_{G}(D)\right)=D$ (see [7]).

Suppose we have case (II) for $B_{1}(11)$. If we put $\chi_{3}(1)=175$, $\chi_{4}(1)=\chi_{5}(1)=1750, \chi_{6}(1)=\bar{\chi}_{6}(1)=126\left(\bar{\chi}_{6}\right.$ is the complex conjugate of $\left.\chi_{6}\right)$, we see that $\chi_{4}(d)=\chi_{5}(d)=0, \chi_{2}(d)=-\chi_{3}(d)$ and hence $\chi_{6}(d)=$ $\overline{\chi_{6}(d)}=1$ for any element $d$ in $G, d$ of order 5 . Now take $d \in D$, and since $\chi_{2}(d) \neq 0$, we have $\chi_{2}(d)=-\chi_{3}(d) \equiv 0(\bmod 5)$.

We now use Result 2 and put $d=x_{1}=x_{2}$ and take $x_{3}$ to be an element of order 11. Since the left-hand side of (2) is nonnegative, $\left|\chi_{2}(d)\right| \leqq 10$, and we get that in all cases, $\left|C_{G}(d)\right| \mid 2^{2} .5^{2}$. As $\langle d\rangle=D$, we have that $0_{5}\left(C_{G}(D)\right)>D$, a contradiction. Hence, case (II) for $B_{1}(11)$ is not possible.

So, throughout the rest of the paper, we are in case (I) for $B_{1}(11)$ and we put

$$
\chi_{2}(1)=3200, \chi_{3}(1)=175, \chi_{4}(1)=1750, \chi_{5}(1)=2520
$$

and $\chi_{6}(1)=\bar{\chi}_{6}(1)=896$.

Since all the characters of $B_{1}(11)$ are rational-valued, except $\chi_{6}$ and $\bar{\chi}_{b}$ which are rational-valued only on 11-regular elements, we obtain 
a congruence modulo 5 for $\chi(x), \chi \in B_{1}(11)$ and $x$ any element of order 5. Put $x=x_{1}=x_{2}$ and $x_{3}=s$, where $s$ is an element of order 11, and equation (2) becomes

$$
\begin{aligned}
a(x, x & ; s)\left|C_{G}(x)\right|^{2} \\
& =|G| \sum_{i=1}^{6} \frac{\left(\chi_{i}(x)\right)^{2} \overline{\chi_{i}(s)}}{\chi_{i}(1)} \\
& =|G|\left(1-\frac{\left(\chi_{2}(x)\right)^{2}}{3200}-\frac{\left(\chi_{3}(x)\right)^{2}}{175}+\frac{\left(\chi_{5}(x)\right)^{2}}{2520}-\frac{\left(x_{6}(x)\right)^{2}}{896}\right) \\
& =2^{2} .5^{2} .11\left(40336-243 \alpha^{2}-29 \gamma^{2}-32 \gamma\right),
\end{aligned}
$$

where $\alpha=\chi_{2}(x)=-\chi_{3}(x), \gamma=\chi_{6}(x)$. Note that $\chi_{5}(x)=\chi_{6}(x)-1=\gamma-1$ by the orthogonality relation. Since the left-hand side of the above formula is nonnegative, we see that

$|\alpha|<15$ and so $\alpha=0, \pm 5$, or +10 , and if

$$
\begin{array}{ll}
\alpha=0, & -34 \leqq \gamma \leqq 36 \\
\alpha= \pm 5, & -34 \leqq \gamma \leqq 31 \\
\alpha= \pm 10, & -24 \leqq \gamma \leqq 21 .
\end{array}
$$

By the orthogonality relation, $1+5 \alpha^{2}+\gamma^{2}+(\gamma-1)^{2} \leqq\left|C_{G}(x)\right|$, and because $\left(\left|C_{G}(x)\right|, 77\right)=1$, we get the following possibilities for any element $x$, of order 5 , in $G$ :

(i) $\alpha=0, \gamma=-4,\left|C_{G}(x)\right| \mid 2^{4} .5^{3}$

(ii) $\alpha=0, \gamma=1,\left|C_{G}(x)\right| \mid 2.3 .5^{2}$

(iii) $\alpha= \pm 5, \gamma=1,\left|C_{G}(x)\right| \mid 2^{2} .3 .5^{2}$.

If $\langle d\rangle=D$ is the defect group of the 5-block $B_{2}(5)$ (of defect 1) which contains $\chi_{2}$, then $\chi_{2}(d)=\alpha \neq 0$, and so $\alpha= \pm 5$ and $\left|C_{G}(d)\right| \mid 2^{2} .3 .5^{2}$. As $0_{5}\left(C_{G}(\langle d\rangle)\right)=D$, it is immediate that $C_{G}(d) / D \cong A_{5}$. It follows now that $C_{G}\left(G_{5}\right)=Z\left(G_{5}\right)$, and that $G_{5}$ is nonabelian and by a result of $\mathrm{B}$. Huppert ([10], S. 8.6), $G_{5}$ is of exponent 5. If $Z\left(G_{5}\right)=\langle\rho\rangle$, where $\rho^{5}=$ 1 , then $\left|C_{G}(\rho)\right| \mid 2^{4} .5^{3}$, and as $\langle\rho\rangle \triangleleft N_{G}\left(G_{5}\right)$, we have by Sylow theorems $\left|N_{G}\left(G_{5}\right)\right|=5^{3}$ or $2^{4} .5^{3}$. The first possibility is impossible by a theorem of H. Wielandt ([10], S. 8.1), and so $N_{G}\left(G_{5}\right)=S . G_{5}$ where $S$ is a 2group of order 16 . Since $Z(S)$ is a cyclic group of order 4 , we have that $N_{G}(\langle d\rangle) \cong F_{20} \times A_{5}$ where $F_{20}$ is a Frobenius group of order 20, and so $d$ is conjugate to all its powers.

We shall now proceed to rule out possibilities (B), (C) and (D) for $B_{1}(7)$. In the cases $(\mathrm{B})$ and $(\mathrm{C})$, we put $x_{1}=x_{2}=d$ and $x_{3}=\nu$, where $\nu^{7}=1$, in equation (2). In both these cases, there are only a small number of possibilities for the values of the characters in $B_{1}(7)$ on the element $d$, and in all cases we get that $\left|C_{G}(d)\right|<2^{2} .3 .5^{2}$, a contradiction; so cases (B) and (C) are not possible for $B_{1}(7)$. Case (D) is immediately ruled out by summing the squares of the degrees so far determined, 
(noting that the 5-block of defect $1, B_{2}(5)$ which contains $\chi_{2}$ has the following degrees: $\left.B_{2}(5)=\{3200,175,825,1925,1925\}\right)$; as this sum is $45,496,297>|G|$.

Hence, for the rest of the paper we are in case (A) for $B_{1}(7)$ and we put $B_{3}(3)$ to be the 3 -block of defect 1 containing the characters $\chi_{7}$ and $\chi_{9}$ of degrees 825 and 1056 respectively. Then

$$
B_{3}(3)=\{825,1056,231\} \text {. }
$$

Let $B_{2}(5)$ be the 5 -block of defect 1 containing $\chi_{2}, \chi_{3}$, and $\chi_{7}$. Then

$$
B_{2}(5)=\{3200,175,825,1925,1925\} \text {. }
$$

Further, we put $\chi_{8}(1)=2750, \chi_{10}(1)=22$ and $\chi_{11}(1)=1408$. Then using Result 2 for $x_{1}=x_{2}=\rho$ and $x_{3}=\nu$, where $\langle\rho\rangle=Z\left(G_{5}\right)$ and $\nu^{7}=1$, we again get a few possibilities for $\chi(\rho), \chi \in B_{1}(7)$, and this gives that $\left|C_{G}(\rho)\right| \mid 2^{2} .5^{3}$. Hence $C_{G}(\rho)$ is precisely of order $4.5^{3}$ and $C_{G}(\rho)$ is a semi-direct product of a cyclic group of order 4 and $G_{5}$. It follows immediately that the Sylow 2-subgroup $S$ of $\mathrm{N}_{G}\left(G_{5}\right)$ is a quasi-dihedral group of order 16 (i.e., $S=\left\langle a, b \mid a^{8}=1=b^{2}, b a b=a^{5}\right\rangle$ ). Now let $l \in G_{5}\langle\rho\rangle$, and $l \chi_{N_{G}\left(G_{5}\right)} d$, then $l$ has precisely 80 conjugates in $N_{G}\left(G_{5}\right)$, and so $C_{G}(l) \cap N_{G}(\langle\rho\rangle)=\langle l\rangle \times\langle\rho\rangle$. Summing the character $\chi_{6}$ on $G_{5}$, we see that $\chi_{6}(l)=1$ and as $C_{G}(\rho) \cap C_{G}(l)=\langle l\rangle \times\langle\rho\rangle$, and $\left|C_{G}(l)\right| \mid 2.3 .5^{2}$, we have $\left|C_{G}(l)\right|=5^{2}$, i.e., $C_{G}(l)=\langle l\rangle \times\langle\rho\rangle$. In particular $l \chi_{G} d$. The 5-structure of $G$ is now completely determined and we summarize these results in the following lemma:

LemMA 1.3. The group $G$ has precisely 3 conjugate classes of elements of order 5 with representatives $d, \rho$ and $l$. A Sylow 5subgroup $G_{5}$ of $G$ is nonabelian of exponent 5 and $N_{G}\left(G_{5}\right)$ is a semidirect product of $G_{5}$ and a quasi-dihedral group of order 16. Also, $Z\left(G_{5}\right)=\langle\rho\rangle$ and $\rho$ has centralizer of order $4.5^{3},\left|C_{G}(l)\right|=5^{2}$, and $C_{G}(d) \cong\langle d\rangle \times A_{5}$. Finally, $N_{G}(\langle d\rangle)$ is isomorphic to the direct product of aFrobenius group of order 20 by $A_{5}$.

2. The 3-structure. Let $\langle c\rangle$ be a Sylow 3-subgroup of $C_{G}(d)$. Then

$$
\begin{aligned}
C_{G}(c) \cap C_{G}(d) & =\langle d\rangle \times\langle c\rangle, N_{G}(\langle c\rangle) \cap C_{G}(d) \\
& \cong S_{3} \times\langle d\rangle, N_{G}(\langle c\rangle) \cap N_{G}(\langle d\rangle) \cong S_{3} \times F_{20}
\end{aligned}
$$

It now follows that $C_{G}(c) \mid\langle c\rangle \cong S_{5} . E$, where $E=\langle 1\rangle$ or $E$ is an elementary 2-group of order 16, and $E \triangleleft C_{G}(c)$. Since $\left.N_{G}(\langle c\rangle)\right\rangle C_{G}(c)$, $G_{3}$ is then elementary abelian; and so $C_{G}(c) \cong\langle c\rangle \times S_{5} \cdot E$.

Suppose $|E|=16$. Let $X$ denote a Sylow 2-subgroup of $N=$ $N_{G}(\langle c\rangle)$, and let $G_{2}$ be a Sylow 2-subgroup (of $G$ ) containing $X$. If $E \triangleleft G_{2}$, then $\left|N_{G}(E): N\right|=2$, and then $2^{4}|| N_{G}(\langle d\rangle) \mid$ (by the Frattini 
argument), clearly a contradiction. We may suppose therefore that $N_{G}(E)=N$. As $X / E$ is not elementary, there is an involution $t \in Z\left(G_{2}\right) \cap E$. It then follows that $\left|C_{G}(t): C_{N}(t)\right|=2$ or 10 , but in either case, $\langle c\rangle \leqq C\left(0_{2}\left(C_{G}(t)\right)\right)$. As $C(E) \cap N=E \times\langle c\rangle,\left|C_{G}\left(G_{3}\right)\right| \mid 2^{3} .3^{2}$. Also, if $0_{2}=0_{2}\left(C_{G}(t)\right)$, then $0_{2} \leqq E$. If $\langle c\rangle$ is a Sylow 3-subgroup of $C\left(0_{2}\right)$, we have a contradiction by the Frattini argument. So a Sylow 3-subgroup $G_{3}$ of $C\left(0_{2}\right)$ is of order 9. By the Frattini argument, $5 \nmid \mid C_{G}(t)$. So $C_{G}(t)$ is a soluble group of order $2^{9} \cdot 3^{2}$. From the structure of $C_{G}(c)$, we must have $\left|0_{2}\right|=4$ but then $G_{3} \triangleleft C_{G}(t)$, which contradicts the fact that $\left|C_{G}\left(G_{3}\right)\right| \mid 2^{3} .3^{2}$ We have proved:

LEMma 2.1. If $\langle c\rangle$ is a Sylow 3-subgroup of $C_{G}(d)$, then $C_{G}(c) \cong$ $\langle c\rangle \times S_{5}$ and $N_{G}(\langle c\rangle) \cong S_{3} \times S_{5}$.

Put $N_{G}(\langle c\rangle)=A \times B$, where $A \cong S_{3}$ and $B \cong S_{5}$. Let $\pi$ be an involution in $A$. Since $A$ is a maximal subgroup of $V$, where $V \cong A_{5}$ and $C_{G}(d)=\langle d\rangle \times V$, it follows that if $\langle\lambda\rangle$ is a Sylow 3-subgroup of $B$, then $C_{G}(\lambda) \cap C_{G}(d)=A$. We have $C_{G}(\pi) \cap N_{G}(\langle d\rangle)=W \times\langle\pi, \tau\rangle$, where $W \cong F_{20}$ and $\langle\pi, \tau\rangle$ is a 4-group. It now follows by Sylow that $\left|C_{G}(\pi)\right|=2^{5} .3 .5,2^{9} .3 .5$ or $2^{6} .3^{2} .5$. In the first case, $\langle\pi, \tau\rangle \triangleleft C_{G}(\pi)$, which contradicts $C_{G}(d) \cap C_{G}(\lambda)$.

If $\left|C_{G}(\pi)\right|=2^{9} .3 .5$, then $0_{2}=0_{2}\left(C_{G}(\pi)\right)$ is elementary abelian of order 64 and $C_{G}(\pi) / 0_{2} \cong S_{5}$. (If $0_{2}$ were nonabelian, then $C_{G}(d) \cap C_{G}(\lambda) \neq\langle\pi\rangle$ ). Let $f$ be an element of order 3 in $C_{G}(d)$ such that $f \in N_{G}(\langle\pi, \tau\rangle)$. Now $N_{G}(\langle\pi, \tau\rangle) \geqq 0_{2} . W$, and $N_{G}(\langle\pi, \tau\rangle) \geqq\langle f\rangle$. Since $C_{G}(\langle\pi, \tau\rangle) \leqq C_{G}(\pi)$, we have that $0_{2}=0_{2}\left(C_{G}(\langle\pi, \tau\rangle)\right)$, and hence $N_{G}\left(0_{2}\right)>C_{G}(\pi)$. However, from Lemma 1.1 and the fact that $A_{7}$ has no elements of order 15, we have that $\left|N_{G}\left(0_{2}\right)\right|=2^{9} .3^{2} .5$; thus $N_{G}\left(0_{2}\right) / 0_{2} \cong\langle f\rangle \cdot S_{5}$ where $0_{2} \cdot\langle f\rangle$ is a normal subgroup of $N_{G}\left(0_{2}\right)$, again contradicting the structure of $C_{G}(d) \cap C_{G}(\lambda)$.

The order of $C_{G}(\pi)$ is thus $2^{6} .3^{2} .5$ and it follows that $C_{G}(\pi) /\langle\pi\rangle \cong$ Aut $\left(A_{6}\right)$. Hence $N_{G}\left(G_{3}\right) / C_{G}\left(G_{3}\right)$ is a semi-dihedral group of order 16 and hence all elements of order 3 are conjugate in $G$.

Let $z$ be the unique involution of a Sylow 2-subgroup $Z$ (of order $4)$ of $C_{G}(\rho)$ where $\langle\rho\rangle=Z\left(G_{5}\right)$. Since $\langle\rho\rangle \chi_{G}\langle d\rangle, z \chi_{G} \pi$, and so $\left|C_{G}(z)\right|=2^{5} .3 .5$ or $2^{9} .3 .5$. In the first case $C_{G}(z) / Z \cong S_{5}$, but since the Sylow 2-subgroup of $N_{G}(\langle\rho\rangle)$ is quasi-dihedral, $z \in \mho^{2}(X)$, where $X$ is a Sylow 2-subgroup of $C_{G}(z)$. However as $X / Z \cong D_{8}$, where $D_{8}$ is a dihedral group of order $8, \mho^{2}(X) \leqq Z$ which gives a contradiction. Hence $\left|C_{G}(z)\right|=2^{9} .3 .5$ and if $E=0_{2}\left(C_{G}(z)\right), E$ is a 2-group of order 64 and $C_{G}(z) / E \cong S_{5}$. Because $C_{G}(\rho) \cap C_{G}(c) \cap C_{G}(z)=Z \cdot\langle\rho\rangle, Z \leqq E$ and $Z \triangleleft C_{G}(z)=C$.

As $\pi \in V$, where $C_{G}(d)=\langle d\rangle \times V$ and $V \cong A_{5}, \pi \notin E$, but $\pi \in V \cdot E$, where $V \cdot E / E \cong A_{5}$. In any case, $N_{c}(\langle c\rangle)=A \times F$, where $A \cong S_{3}$ and 
$F \cong D_{8}$, with $F \cap E=Z$. Thus $C_{C}(\pi) \cdot E$ is a Sylow 2-subgroup of $C=C_{G}(z)$, and hence $\left|C_{E}(\pi)\right| \leqq 8$.

If $E$ is abelian, it is of type $(4,2,2,2,2)$ and

$$
C_{G}(c) \cap E=C_{G}(\rho) \cap E=Z \text {. Also, }\left|C_{G}(\pi) \cap \Omega_{1}(E)\right| \geqq 8,
$$

and as $C_{G}(\pi) \geqq Z$, we have a contradiction. Hence $E$ is nonabelian and thus $E$ is a central product of two quaternion groups $Q_{1}$ and $Q_{2}$, and the cyclic group $Z$ of order 4 . We have proved:

LEMmA 2.2. The group $G$ has only ond conjugate class of elements of order $3, C_{G}\left(G_{3}\right)=\langle\pi\rangle \times G_{3}$, where $\pi$ is an involution, and $N_{G}\left(G_{3}\right) /$ $C_{G}\left(G_{3}\right)$ is a semidihedral group of order 16 . If $z$ is the involution in $C_{G}(\rho)$, where $\langle\rho\rangle=Z\left(G_{5}\right)$, then $C=C_{G}(z)$ is an extension of $a$ nonabelian 2-group $E$ of order 64, (which is a central product of two quaternion groups and a cyclic group of order four) by the symmetric group $S_{5}$ on 5 letters. Finally, $C_{G}(\pi) /\langle\pi\rangle \cong \operatorname{Aut}\left(A_{6}\right)$.

3. Determination of all degrees of irreducible characters of $G$. We are now in a position to apply the exceptional character theory to the group $G$ with respect to the subgroup $H=N_{G}(\langle c\rangle)$, where $c$ is any element of order 3. As "special classes" (in the sence of Wong [15]), we take all roots of $c$. As $H \cong S_{3} \times S_{5}$, the character table of $H$ is determined from the character tables of $S_{3}$ and $S_{5}$. Put $H=$ $A \times B$, where $A \cong S_{3}$ and $B \cong S_{5}$.

In the above notation, our special classes of $H$ are the conjugate classes in $H$ with representatives $c, c t, c z, c w$ and $c d$. As usual, $B_{\tau}(3)$ will denote a 3-block of $G$ and $b_{\tau}(3)$, a 3-block of $H$. The group $H$

Character Table of $A \cong S_{3}$

\begin{tabular}{|c|c|c|r|r|}
\hline Order & Element & $\theta_{1}$ & $\theta_{2}$ & \multicolumn{1}{|c|}{$\theta_{3}$} \\
\hline 1 & 1 & 1 & 1 & 2 \\
2 & $\pi$ & 1 & -1 & 0 \\
3 & $c$ & 1 & 1 & -1 \\
\hline
\end{tabular}

Character Table of $B \cong S_{5}$

\begin{tabular}{|c|r|r|r|r|r|r|r|r|}
\hline Order & Element & $\zeta_{1}$ & \multicolumn{1}{|c|}{$\zeta_{2}$} & \multicolumn{1}{c|}{$\zeta_{3}$} & \multicolumn{1}{c|}{$\zeta_{4}$} & \multicolumn{1}{|c|}{$\zeta_{5}$} & \multicolumn{1}{|c|}{$\zeta_{6}$} & \multicolumn{1}{c|}{$\zeta_{7}$} \\
\hline 1 & 1 & 1 & 1 & 4 & 4 & 6 & 5 & 5 \\
2 & $z$ & 1 & 1 & 0 & 0 & -2 & 1 & 1 \\
2 & $t$ & 1 & -1 & -2 & 2 & 0 & -1 & 1 \\
4 & $w$ & 1 & -1 & 0 & 0 & 0 & 1 & -1 \\
3 & $\lambda$ & 1 & 1 & 1 & 1 & 0 & -1 & -1 \\
6 & $\lambda t$ & 1 & -1 & 1 & -1 & 0 & -1 & 1 \\
5 & $d$ & 1 & 1 & -1 & -1 & 1 & 0 & 0 \\
\hline
\end{tabular}


has three 3-blocks:

$$
\begin{aligned}
& b_{1}(3)=\left\{1_{I I}, \theta_{2}, \theta_{3}, \zeta_{B}, \zeta_{3} \theta_{2}, \zeta_{3} \theta_{3}, \zeta_{6}, \zeta_{6} \theta_{2}, \zeta_{6} \theta_{3}\right\} \\
& b_{2}(3)=\left\{\zeta_{2}, \zeta_{2} \theta_{2}, \zeta_{2} \theta_{3}, \zeta_{4}, \zeta_{4} \theta_{2}, \zeta_{4} \theta_{3}, \zeta_{7}, \zeta_{7} \theta_{2}, \zeta_{7} \theta_{3}\right\} \\
& b_{3}(3)=\left\{\zeta_{5}, \zeta_{5} \theta_{2}, \zeta_{5} \theta_{3}\right\} .
\end{aligned}
$$

Here $1_{H I}=\theta_{1} \cdot \zeta_{1}$, and $b_{1}(3), b_{2}(3)$ are the 3 -blocks of defect 2 of $H$; and $b_{3}(3)$ the unique 3 -block of defect 1 of $H$. We denote by $b_{-}(3)^{G}$, the block of $G$ which corresponds to the block $b_{-}(3)$ of $H$, using Brauer's block correspondence (see [4]). By [15], Theorem 6 (or [4], S.2E)

$$
\begin{aligned}
& b_{1}(3)^{G}=B_{1}(3) \\
& b_{2}(3)^{\sigma}=B_{2}(3) \\
& b_{3}(3)^{G}=B_{3}(3)=\{825,1056,231\},
\end{aligned}
$$

where $B_{2}(3)$ is the only other 3-block of defect 2 of $G$ besides the principal 3-block $B_{1}(3)$. If $D$ denotes the union of special classes of $H$, we take the following basis for the module of all generalized characters of $H$ which vanish on $H \backslash D$ :

$$
\begin{aligned}
& \varphi_{1}=\left(1_{H}-\zeta_{3}\right) \Sigma \\
& \varphi_{2}=\left(1_{H}+\zeta_{6}\right) \Sigma, \\
& \varphi_{3}=\left(\zeta_{2}-\zeta_{4}\right) \Sigma, \\
& \varphi_{4}=\left(\zeta_{2}+\zeta_{7}\right) \Sigma,
\end{aligned}
$$

and

$$
\varphi_{5}=\zeta_{5} \Sigma
$$

where

$$
\Sigma=1_{I I}+\theta_{2}-\theta_{3} \text {. }
$$

Note that $\varphi_{1}$ and $\varphi_{2}$ are expressed as a linear combination of irreducible characters occurring only in $b_{1}(3)$. Similarly $\varphi_{3}$ and $\varphi_{4}$; and $\varphi_{5}$ are expressed only as a linear combination of characters occurring in $b_{2}(3)$, and $b_{3}(3)$ respectively. Let $\varphi_{i}^{*}$ denote the corresponding induced characters of $\phi_{2}(i=1,2, \cdots, 5)$.

The induced characters $\varphi_{1}^{*}, \varphi_{2}^{*}$ can be expressed as a linear combination of the irreducible characters of $B_{1}(3)$ and if $\chi \in B_{1}(3)$, then $\chi$ appears as a constituent of $\varphi_{1}^{*}$ or $\varphi_{2}^{*}$ (see [15], Ths. 7 and 9). Similar statements can be made for $\varphi_{3}^{*}, \varphi_{4}^{*}$ and $B_{2}(3)$, and $\varphi_{5}^{*}$ and $B_{3}(3)$.

Finally, if $\chi$ is any irreducible character of $G$, let $n_{i}=\left(\chi, \varphi_{i}^{*}\right)$, $(i=1,2, \cdots, 5)$. Then

$$
\begin{aligned}
\chi(\sigma)= & -n_{1} \zeta_{3}(\sigma)+n_{2} \zeta_{6}(\sigma)-n_{3} \zeta_{4}(\sigma)+n_{4} \zeta_{7}(\sigma) \\
& +n_{5} \zeta_{5}(\sigma), \text { for any } \sigma \in D .
\end{aligned}
$$


Since $\left(\varphi_{i}^{*}, \varphi_{j}^{*}\right)_{G}=\left(\varphi_{i}, \varphi_{j}\right)_{H}$ we have:

$$
\begin{aligned}
& \left(\varphi_{1}^{*}, \varphi_{1}^{*}\right)=\left(\varphi_{2}^{*}, \varphi_{2}^{*}\right)=\left(\varphi_{3}^{*}, \varphi_{3}^{*}\right)=\left(\varphi_{4}^{*}, \varphi_{4}^{*}\right)=6, \\
& \left(\varphi_{1}^{*}, \varphi_{2}^{*}\right)=\left(\varphi_{3}^{*}, \varphi_{4}^{*}\right)=3, \\
& \left(\varphi_{1}^{*}, \varphi_{3}^{*}\right)=\left(\varphi_{2}^{*}, \varphi_{3}^{*}\right)=\left(\varphi_{1}^{*}, \varphi_{4}^{*}\right)=\left(\varphi_{2}^{*}, \varphi_{4}^{*}\right)=0, \\
& \left(\varphi_{5}^{*}, \varphi_{3}^{*}\right)=3
\end{aligned}
$$

and $\left(\varphi_{5}^{*}, \varphi_{i}^{*}\right)=0$ for $i=1,2,3,4$.

Further, by the Frobenius reciprocity law,

$$
\left(\varphi_{1}^{*}, 1_{G}\right)=\left(\varphi_{2}^{*}, 1_{G}\right)=1 \text { and }\left(\varphi_{3}^{*}, 1_{G}\right)=\left(\varphi_{4}^{*}, 1_{G}\right)=0 \text {. }
$$

From these values, it follows that

$$
\left\{\begin{array}{l}
\varphi_{1}^{*}=1_{G}+\sum_{i=1}^{5} \varepsilon_{i} X_{i} \\
\text { and } \\
\varphi_{2}^{*}=1_{G}+\varepsilon_{1} X_{1}+\varepsilon_{2} X_{2}+\eta_{1} Y_{1}+\eta_{2} Y_{2}+\eta_{3} Y_{3}
\end{array}\right.
$$

where $X_{i}$ and $Y_{i}$ are distinct non-principal irreducible characters of $G$.

So far, the degrees of 15 irreducible characters of $G$ have been determined:

\begin{tabular}{|c|c|c|c|c|c|c|c|c|}
\hline$\chi_{1}=1_{G}$ & $\chi_{2}$ & $\chi_{3}$ & $\chi_{4}$ & $\chi_{5}$ & $\chi_{6}$ & $\bar{\chi}_{6}=\chi_{7}$ & $\chi_{8}$ & $\chi_{9}$ \\
\hline 1 & 3200 & 175 & 1750 & 2520 & 896 & 896 & 825 & 1056 \\
\hline
\end{tabular}

\begin{tabular}{|c|c|c|c|c|c|}
\hline$\chi_{10}$ & $\chi_{11}$ & $\chi_{12}$ & $\chi_{13}$ & $\chi_{14}$ & $\chi_{15}$ \\
\hline 2750 & 22 & 1408 & 231 & 1925 & 1925 \\
\hline
\end{tabular}

Using (3) and the fact that $\left(\left.\chi\right|_{G_{3}}, 1_{G_{3}}\right)$ is an integer where $G_{3}$ is a Sylow 3-subgroup of $G$, we get $\chi_{13}(c)=6$ and so

$$
\varphi_{5}^{*}=\chi_{13}+\chi_{8}-\chi_{9} \text {. }
$$

It follows that $\chi_{2}(c d)=\chi_{8}(c d)=-\chi_{3}(c d)=-\chi_{14}(c d)=-\chi_{15}(c d)=1$. Further, $\chi_{11}(c)=4$ and hence, if $\chi_{11} \in B_{1}(3)$ then $\left(\varphi_{1}^{*} \cdot \chi_{11}\right)=-1$ and $\left(\varphi_{2}^{*}, \chi_{11}\right)=0$. By obtaining a congruence modulo 9 for the above characters on the element $c$, we see that if $\chi_{i} \in B_{1}(3)$, then $\chi_{i}$ occurs in precisely one of $\varphi_{1}^{*}$ or $\varphi_{2}^{*}$ if $i=2,3,4,6,7,10,11$ and 12; and in both $\varphi_{1}^{*}$ and $\varphi_{2}^{*}$ if $i=1,14$ and 15, using the block-intersection lemma of Brauer-Tuan ([6], Lemma 3), and since $\chi_{2}(c d)=1$, we have the following possibilities for $B_{1}\left(3_{1}\right), B_{2}(3)$ :

(a) $B_{1}(3)=\{1,3200,1750,22,1408,2750, \cdots\}$, $B_{2}(3)=\{175,896,896, \cdots\}$

(b) $B_{1}(3)=\{1,3200,175,1750,896,896,22,1408,2750\}$, 


$$
\begin{aligned}
& B_{2}(3)=\{1925, \cdots\} \\
& \text { (c) } B_{1}(3)=\{1,1750,896,896,2750,1408, \cdots\}, \\
& B_{2}(3)=\{3200,175,22, \cdots\} \\
& \text { (d) } B_{1}(3)=[1,1750,896,896,2750,22, \cdots\}, \\
& B_{2}(2)=\{3200,175,1408, \cdots\} .
\end{aligned}
$$

Note that $\chi_{6}$ and $\chi_{7}$ both lie in the same block, and

$$
\left(\varphi_{i}^{*}, \chi_{6}\right)=\left(\varphi_{i}^{*}, \chi_{7}\right),(i=1, \cdots, 4)
$$

as $\varphi_{i}^{*}$ is rational-valued. Further, $\chi_{4}(c d)=\chi_{10}(c d)=0$ and since both $\chi_{4}, \chi_{10}$ always lie in $B_{1}(3), \chi_{4}$ and $\chi_{10}$ must be constituents of $\varphi_{2}^{*}$; and as $\chi_{6}(c d)=\chi_{7}(c d)=1, \chi_{6}$ and $\chi_{7}$ either both occur in $\varphi_{1}^{*}$ or both in $\varphi_{3}^{*}$.

By summing the squares of the degrees so far determined, any other irreducible character of $G$ must have degree $77 k$, where $k \leqq 20$. In particular, if $\chi \in B_{1}(3)$ or $B_{2}(3)$, then $(k, 3)=1$ and $k \leqq 20$.

Case (b) for $B_{1}(3)$ and $B_{2}(3)$ is immediately ruled out using (4).

In case (c) for $B_{1}(3)$ ahd $B_{2}(3)$, first assume that neither $\chi_{14}$ nor $\chi_{15}$ appears in $\varphi_{1}^{*}$. Then $\varphi_{3}^{*}(1)=3200-175-22-1925+847=0$. But $847=7 \times 11^{2}$ which is not possible. Hence either $\chi_{14}$ or $\chi_{15}$ appears in $\varphi_{1}^{*}$ with nonzero multiplicity and we have:

$$
\begin{aligned}
& \varphi_{1}^{*}(1)=1+896+896-1408-1925+1540=0, \\
& \varphi_{2}^{*}(1)=1-1705+2750-616-1925+1540=0, \\
& \varphi_{3}^{*}(1)=3200-175-22-1925+\delta_{1} z_{1}+\delta_{2} z_{2}=0,
\end{aligned}
$$

and

$$
\varphi_{4}^{*}(1)=-1925+\delta_{1} z_{1}+\delta_{2} z_{2}+\sum_{i=1}^{3} \kappa_{i} y_{i}=0,
$$

where $\delta_{1}, \delta_{2}, \kappa_{i}(i=1,2,3)$ are equal to \pm 1 and $z_{1}, z_{2}, y_{1}, y_{2}, y_{3}$ are degrees of irreducible characters of $G$. No matter what values the $z_{i}$ takes, at least two of the $y_{i}$ take the value 1232 or one of them takes the value 1540. In any case, the sum of the squares of the degrees so far determined is greater than $|G|$.

In case (d) for $B_{1}(3)$ and $B_{2}(3)$, we have;

$$
\begin{aligned}
& \varphi_{1}^{*}(1)=1+896+896-22-1925+154=0, \\
& \varphi_{2}^{*}(1)=1-1750+2750+770-1925+154=0, \\
& \varphi_{3}^{*}(1)=3200-175-1408-1925+\sum_{i=1}^{2} \delta_{i} z_{i}=0,
\end{aligned}
$$

and

$$
\varphi_{4}^{*}(1)=-1925+\sum_{i=1}^{2} \delta_{i} z_{i}+\sum_{i=1}^{3} \kappa_{i} y_{i}=0,
$$

in the same notation as above. We may take $\left(\varphi_{1}^{*}, \chi_{14}\right)=-1$, and then 
on the element $c t$ of order 6 , we get:

$$
\chi_{2}(c t)=-2, \chi_{3}(c t)=2, \chi_{8}(c t)=0, \chi_{14}(c t)=-1
$$

and $\chi_{15}(c t)=1$. Summing over the 5-block of defect $1, B_{2}(5)$, and using [1], Corollary 4, we get

$$
-2+0=-2 \neq 2+1-1=2,
$$

and a contradiction and so case (a) is the only possibility for $B_{1}(3)$ and $B_{2}(3)$.

In case (a), we have:

$$
\begin{aligned}
& \varphi_{1}^{*}(1)=1+3200-22-1408-1925+154=0, \\
& \varphi_{2}^{*}(1)=1-1750+2750+770-1925+154=0, \\
& \varphi_{3}^{*}(1)=896+896-175-1925+\sum_{i=1}^{2} \delta_{i} z_{i}=0,
\end{aligned}
$$

and

$$
\varphi_{4}^{*}(1)=-1925+\sum_{i=1}^{2} \delta_{i} z_{i}+\sum_{j=1}^{3} \kappa_{i} y_{j}=0 \text {. }
$$

Thus $\sum_{i=1}^{2} \delta_{i} z_{i}=308=4 \times 77$ and $\sum_{j=1}^{3} \kappa_{j} y_{j}=1617=21 \times 77$, which gives a number of possibilities for $z_{i}$ and $y_{j}$. However, if

$$
\chi \notin B_{2}(3) \cup B_{2}(3) \cup B_{3}(3) \text {, then } 9 \times 77 \mid \chi(1) .
$$

By summing the squares of degrees and using this fact, we get a unique decomposition for $\varphi_{3}^{*}$ and $\varphi_{4}^{*}$ :

$$
\varphi_{3}^{*}(1)=896+896-175-1925+154+154=0,
$$

and

$$
\varphi_{4}^{*}(1)=770+770+77-1925+154+154=0 .
$$

There are now either 2 or 5 irreducible characters left to be determined (in the first case $G$ would have one irreducible character of degree 693 and one of degree 1386: while in the second possibility $G$ would have five irreducible characters of degree 693); and so $G$ has either 24 or 27 irreducible characters. Using the orthogonality relations for the element $l$ of order 5 (see Lemma 1.3) and with centralizer, $C_{G}(l)$ of order 25 , it follows immediately that $G$ has only 24 irreducible characters and hence 24 conjugate classes of elements. The character table of $G$ can now be completed, except for some classes of 2-elements which have not as yet been determined.

The partially completed character table of $G$ shows that the characters $\chi_{6}$ ahd $\chi_{7}$ vanish on all 2-elements except the involution $\pi$ 
(defined in Lemma 2.2). A result of Frobenius and Schur (see [8], (3.5)) shows that $\pi$ is not the square of any element of order four in $G$.

4. Completion of proof of theorem. Let $z$ be the central involution with centralizer $C_{G}(z)=C$, as in Lemma 2.2. Then $C / 0_{2}(C) \cong$ $S_{5}$, and $0_{2}(C)$ is a central product of two quaternion groups $Q_{1}$ and $Q_{2}$ and a cyclic group $Z$ of order four. We may take $\langle c\rangle$ and $P=\langle\rho\rangle$ to be a Sylow 3- and Sylow 5-subgroup of $C$ respectively (for definitions of $\langle c\rangle$ and $\langle\rho\rangle$, see Lemmas 1.3 and 2.1). Let $E=0_{2}(C)$, then

$$
C_{C}(c) \cap E=C(P) \cap E=Z,\left|N_{C}(P)\right|=2^{4} .5
$$

with $N_{C}(P)$ having as a Sylow 2-subgroup a quasidihedral group of order 16; and $N_{C}(\langle c\rangle) \cong S_{3} \times D_{8}$, where $D_{8}$ is a dihedral group of order 8. The action of $P$ and $\langle c\rangle$ on $E$ shows that $\left|C: C^{\prime}\right|=2$ and hence $C^{\prime} \mid E \cong A_{5}$. We may suppose that the involution $\pi$ lies in $C^{\prime} \backslash E$. From the structure of $C_{G}(c)$, if $Z=\langle\omega\rangle$, where $\omega^{4}=1$, then $C_{G}(\omega)=C^{\prime}$. Further, if $u$ is any element of order four in $E \backslash Z$, then $\left|C_{G}(u)\right|=2^{8}$; as $u$ must have exactly 30 conjugates in $C$ and as $\mho^{1}(E)=\langle z\rangle$. Similarly, if $t$ is an involution in $E Z$, then $\left|C_{C}(t)\right|=2^{8}$.

So far, we have determined the order of 20 of the 24 conjugate classes of elements of $G$. If $K_{1}, K_{2}, K_{3}$ and $K_{4}$ denote the remaining four classes of 2-elements of $G$, then using the previous lemmas and summing the order of the conjugate classes so far determined, we have the following possibilities:

$$
\left|C_{G}\left(x_{1}\right)\right| \quad\left|C_{G}\left(x_{2}\right)\right| \quad \mid C_{C}
$$

(3) $2^{6} \quad 2^{3}$

$2^{3}$

$2^{4}$

$2^{3}$

where $x_{i} \in K_{i}, i=1,2,3,4$.

It now follows that for $t \in E \backslash Z, t$ an involution, then $t \widetilde{G} z$. If $x$ is an involution in $C^{\prime} \backslash E$, then by Sylow theorems, we may take $x \in N(P) \cap C^{\prime}$. However the Sylow 2-subgroup of $N_{C^{\prime}}(P)$ contains only two involutions $x$ and $x z$ which are conjugate in $N_{C}(P)$, and so $x \widetilde{G} \pi$; and hence $C^{\prime} \backslash E$ has only one class of involutions. A transfer theorem of J. G. Thompson ([13], Lemma 5.38) now shows that $G$ has precisely two conjugate classes of involutions with representatives $z$ and $\pi$.

As before, we may take $\pi \in N_{C^{\prime}}(\langle c\rangle) \backslash E \cap N(\langle c\rangle)$. Put $N_{C}(\langle c\rangle)=$ $\langle c\rangle \cdot\langle\pi\rangle \times K$, where $K$ is a dihedral group of order 8 . Denote the involutions of $K$ by $z, \tau, \tau z, \tau \omega^{-1}$ and $\tau \omega$, where $Z=\langle\omega\rangle$ is as above and $Z$ is the unique cyclic group of order four in $K$. From the structure of $C_{G}(c)$, we may take $\tau \widetilde{G}_{\widetilde{G}} \tau z_{\widetilde{G}} z$ and $\pi \widetilde{G}_{\tau} \tau \omega_{\widetilde{G}} \tau \omega^{-1}$. Further, $[\langle c\rangle, E]$ is an extra-special 2-group of order 32 and we write $[\langle c\rangle, E]=Q_{1} Y Q_{2}$, where $Q_{1}$ and $Q_{2}$ are quaternion groups of order 8 
and $Q_{1} Y Q_{2}$ denotes the central product of $Q_{1}$ and $Q_{2}$. Put $Q_{1}=\left\langle\alpha_{1}, \alpha_{2}\right\rangle$, $Q_{2}=\left\langle\alpha_{2}, \alpha_{4}\right\rangle\left(\alpha_{i}, i=1, \cdots, 4\right.$ are elements of order 4) and note that $\langle\pi\rangle \cdot K \leqq N_{C}\left(Q_{1} Y Q_{2}\right)$.

As $\left|C_{E}(\pi)\right| \leqq 8$, we may choose the $\alpha_{i}$ in such a way that $\alpha_{1}^{\pi}=$ $\alpha_{1}^{-1}, \alpha_{2}^{\pi}=\alpha_{2}^{-1}, \alpha_{3}^{\pi}=\alpha_{1} \alpha_{3}$ and $\alpha_{4}^{\pi}=\alpha_{2} \alpha_{4}$. Hence $C_{E}(\pi)$ is an abelian group of order 8 and type $(4,2)$, and $C_{E}(\pi)=Z \times\left\langle\alpha_{1}, \alpha_{2}\right\rangle$. Similarly, as $c \in C_{G}(\tau)$ and $\tau \in N_{C}\left(Q_{1} Y Q_{2}\right)$; and $\tau \omega_{\widetilde{G}} \pi$, we get $\alpha_{1}^{\tau}=\alpha_{2}$ and $\alpha_{3}^{\tau}=\alpha_{4}$ (again with appropriate choice of the $\alpha_{i}$ ). Since $\pi$ is not the square of any elemement of order four, an easy computation now gives that $\mho^{1}\left(C_{c}(\pi)\right)=\left\langle\omega \alpha_{1} \alpha_{2} \pi\right\rangle$. A result of Gaschutz ([10], S. 17.4) shows that $C_{G}(\pi) \cong\langle\pi\rangle \times \operatorname{Aut}\left(A_{6}\right)$.

If we put $v=\omega \pi$, then $v$ is of order four and $C_{C^{\prime}}(v)=C_{C^{\prime}}(\pi)$. Certainly, $v^{2}=z$ and as $\alpha_{1} \tau \in C_{c}(v)$, we have that $\left|C_{C}(v)\right|=\left|C_{G}(v)\right|=2^{6}$. The action of $\pi$ on $E$ gives that the coset $E \pi$ has precisely eight elements of order four whose square is $z$, and so $C^{\prime} \backslash E$ contains one conjugate class of elements of order four (with representative $v$ ) whose square is $z$. The action of $\tau$ and $c$ on $E$ shows that $C \backslash C^{\prime}$ has no element of order four whose square is $z$.

The group $G$ has therefore precisely three conjugate classes of elements of order four, with representatives $\omega, u$ and $v$.

The orthogonality relations enable us to complete the character table on all but the last three classes of $G$. It can be shown by an easy computation that there are no elements of order 16 . In any case, from the orthogonality relations the character $\chi_{11}$ of degree 22 vanishes on the remaining 3 conjugate classes of 2-elements.

We give below only part of the character table of $G$, namely, the value of the irreducible character $\chi_{11}$ of degree 22 on certain elements. Note that for any element $x$ of order eight, $\chi_{11}(x)=0$, from the orthogonality relation.

\begin{tabular}{|l|r|r|}
\hline Element $(s)$ & Order & \multicolumn{1}{|c|}{$\chi_{11}$} \\
\hline 1 & 1 & 22 \\
$c$ & 3 & 4 \\
$c z$ & 6 & 0 \\
$\rho$ & 5 & -3 \\
$l, d$ & 5 & 2 \\
$z$ & 2 & 6 \\
$w$ & 4 & -6 \\
$u, v$ & 4 & 2 \\
$x$ & 8 & 0 \\
\hline
\end{tabular}

Using the same notation as above, $C_{E}(\tau)=\left\langle\alpha_{1} \alpha_{2}, \alpha_{3} \alpha_{4}, z\right\rangle$, an elementary abelian group of order 8 . If we put $F=\langle\tau\rangle \times C_{E}(\tau)$, then $F$ is an elementary abelian group of order 16 , and further, $\langle E, c, \pi\rangle \leqq$ 
$N_{C}(F)$. Hence $F$ contains precisely one class of involutions in $G$ (since $\tau \widetilde{G}_{z} z$ ) and $\left|N_{C}(F)\right|=2^{8} .3$, as $E \cap F \triangleleft C$. Also, $C_{G}(F)=C_{C}(F)=F$.

The coset $E \tau$ contains precisely 16 involutions, and so, if $\kappa$ is an involution in $C \backslash C^{\prime}$, then $\kappa_{\widetilde{c}} \tau$ or $\kappa_{\widetilde{c}} \tau \omega$. Now, let $J$ be any elementary abelian subgroup of order 16 of $C$, such that $J$ contains only involutions conjugate to $z$. Then $|J \cap E|=8$, and if $\kappa \in J \backslash E$, then $\kappa \in C \backslash C^{\prime}$ and hence $\kappa \widetilde{c} \tau$. Thus $C$ has precisely one class of elementary abelian subgroup of order 16 containing only involutions conjugate to $z$. The structure of $A_{8}$ gives the following result:

LEMmA 4.1. If $F$ is an elementary abelian subgroup of order 16 in $C$, and if all involutions in $F$ are conjugate to $z$, then $N_{G}(F) \backslash F \cong S_{6}$.

Clearly, $z$ has 15 conjugates in $N_{G}(F)$, and so $N_{G}(F)^{\prime}=M$ is of index 2 in $N_{G}(F)$, and $M \backslash F \cong A_{6}$. Since $\langle c\rangle \leqq M$ and $\left|C_{M}(c) \cdot F \backslash F\right|=$ 9, $\omega \notin M$. Thus $|E: M \cap E|=2^{5}$ and there must be an involution $t \in E \cap M \backslash F \cap E$. Further, $\left|C_{F}(t)\right|=4$ and as the normalizer of a Sylow 5-subgroup of $M$ is dihedral of order 10, there must be precisely one class of involutions in $M \backslash F$ and they are all conjugate to $z$ in $G$.

If $\mu$ is an element of order five in $M$, then $N_{M}(\langle\mu\rangle)=\langle\mu\rangle\langle i\rangle$, for some involution $i$ in $M \backslash F$. Since $i_{\widetilde{G}} z, \mu$ must be conjugate to $l$ or $d$ in $G$, since if $\mu_{\widetilde{G}} \rho$, then $z \widetilde{G} \pi$.

Since $c$ normalizes $[c, E] \cap M$, and

$$
|[c, E] \cap M| \geqq 16,[c, E]=E \cap M .
$$

As $|\langle\pi, \omega\rangle \cap M|=4$, and $\pi, \omega \notin M,\langle\pi \omega\rangle=\langle\pi, \omega\rangle \cap M$, and $\langle\pi \omega\rangle$. $[c, E] \cdot F / F \cong D_{8}$ and is a Sylow 2-subgroup of $M / F$. Let $T / F=$ $\langle\pi \omega\rangle \cdot[c, E] \cdot F / F$. Since the action of $\pi, \tau$ on $E$ has been completely determined above, the action of $T / F$ on $F$ is now completely determined. If we regard $F$ as a vector space with basis $z, \tau, \alpha_{1} \alpha_{2}$ and $\alpha_{3} \alpha_{4}$, the action of $T$ on $F$ is given by:

$$
t_{0}=\alpha_{1} \alpha_{3} \alpha_{4} z=\left[\begin{array}{cccc}
1 & 0 & 0 & 0 \\
1 & 1 & 1 & 0 \\
0 & 0 & 1 & 0 \\
1 & 0 & 0 & 1
\end{array}\right], \quad t_{1}=\alpha_{4} \alpha_{1} \alpha_{2}=\left[\begin{array}{cccc}
1 & 0 & 0 & 0 \\
1 & 1 & 0 & 1 \\
1 & 0 & 1 & 0 \\
0 & 0 & 0 & 1
\end{array}\right]
$$

and

$$
t_{2}=\pi \omega \tau=\left[\begin{array}{llll}
1 & 0 & 0 & 0 \\
1 & 1 & 0 & 0 \\
0 & 0 & 1 & 0 \\
0 & 0 & 1 & 1
\end{array}\right]
$$


where $t_{0}, t_{1}$ and $t_{2}$ are involutions, $\left\langle t_{0}, t_{1}, t_{2}\right\rangle \cong D_{8}$ where $D_{8}$ is a dihedral group of order 8 with $Z\left(\left\langle t_{0}, t_{1}, t_{2}\right\rangle\right)=\left\langle t_{0}\right\rangle$ and $T=\left\langle t_{0}, t_{1}, t_{2}\right\rangle \cdot F$. Note that $\left(t_{1} t_{2}\right)^{2}=t_{0}$ and $\left|C_{M}\left(t_{1} t_{2}\right)\right|=2^{3}$, and $\left\langle\tau t_{1} t_{2}\right\rangle$ of order 8 is selfcentralizing in $M$. As $\left|C_{M}\left(t_{0}\right): C_{M}\left(t_{1} t_{2}\right)\right|=4, t_{1} t_{2} \nsim \omega$, also if $x$ is any element of order four such that $x^{2} \in F$, then $x \not^{G} \omega$ as $\omega \notin M$.

The group $M$ has therefore 195 involutions (all conjugate to $z$ in $G), 1260$ elements of order four, all conjugate in $G$ to either $u$ or $v$, 720 elements of order eight, 800 elements of order three, 480 elements of order six and 2,304 elements of order five. Summing $\chi_{11}$ over the group $M$, we find that $\left(\chi_{11}, 1\right)_{M}=2$. Let $U=V \cdot G_{3}$ be a Sylow 3normalizer in $M$, where $V$ is a cyclic group of order four and $G_{3}$ is a Sylow 3-subgroup of $M$. By an easy computation, $\left(\chi_{11}, 1\right)_{U}=4$. Now take a quaternion group $W$ of order eight, $W \leqq N_{G}\left(G_{3}\right)$, and $V<W$. Since $C \backslash C^{\prime}$ contains no elements of order four whose square is $z, W$ contains only elements of order four which are conjugate to $u$ or $v$. If $L=W \cdot G_{3}$, then we find that $\left(\chi_{11}, 1\right)_{L}=3$. Since $\left.2+3\right\rangle 4,\langle L, M\rangle=$ $H$ is a proper subgroup of $G$, and $|H|=2^{7} \cdot 3^{2} \cdot 5 . k$, where $k$ is an integer, $k>1$.

If $N$ is a normal subgroup of $H$ and $1<N \leqq M$, then $N=F$ or $N=M$. Since $H=N_{G}(F)$ contradicts the structure of $S_{6},|H: M|>$ 10. From the degrees of the irreducible characters of $G$, we see that if $\bar{G}$ is a subgroup of $G$ with $|G: \bar{G}| \leqq 200$, then $|\bar{G}|=2^{7} \cdot 3^{2}$.5.7.11, $2^{5} .3^{2} .5^{3} .7$ or $2^{9} .5^{2} .7 .11$; that is $|G: \bar{G}|=100,176$ or 45 respectively.

The following are therefore the only possibilities for $|H|$ : (a) $2^{7} .3^{2} .5^{3}$, (b) $2^{7} .3^{2} \cdot 5^{2} .7$, (c) $2^{7} .3^{2} \cdot 5.11$, $2^{8} .3^{2} .5 .7$, (g) $2^{9} .3^{2} \cdot 5^{2}$, (h) $2^{9} \cdot 3^{2} \cdot 5.7$.

(d) $2^{7} \cdot 3^{2} \cdot 5.7 .11$, (e) $2^{8} \cdot 3^{2} \cdot 5.11$,

In the cases (a) to (d), $H$ must be a simple group and a result of Z. Janko [11], shows that we are in case (d) and then $H \cong M_{22}$. Cases (f) and (g) can be eliminated by Sylow theorems and the structure of the Sylow 7- and 11-normalizers. If we are in case (g), we may take $z$ to be a central involution in $H$ and then $N_{H}(F)=N_{G}(F)$. However, now $\left|H: N_{H}(F)\right|=10$ which contradicts the order of $A_{10}$. Finally, suppose we are in case (h). If $H_{5}$ is a Sylow 5-subgroup of $H$, then as $H$ is simple by the Frattini argument $\left|N_{G}\left(H_{5}\right)\right|=2^{4} .5^{2}$. This is a contradiction, as no subgroup of order $5^{2}$ has normalizer divisible by 16 .

We have thus shown that case (d) is the only possibility for the order of $H$ and so $H$ is isomorphic to the Mathieu simple group $M_{22}$. The degrees of the irreducible characters of $G$ yield the following result:

LEMMA 4.2. The group $G$ is a primitive permutation group of degree 100 with stabilizer of a point $H$ isomorphic to the Mathieu 
simple group $M_{22}$ and the orbits of $H$ are of length 1,22 and 77.

With Result 3 and Lemma 4.2, the theorem is proved.

The authors would like to thank Professor D. Wales for his kind permission to quote Result 3.

\section{REFERENCES}

1. R. Brauer, Investigations on group characters, Ann. of Math. (2) 42 (1941), 936-958.

2. - On groups whose order contains a prime to the first power, I, Amer. J. Math. 64 (1942), 401-420.

3. - Zur Darstellungstheorie der Gruppen endlicher Ordnung, I, Math. Zeit. 63 (1956), 406-444.

4. — Zur Darstellungstheorie der Gruppen endlicher Ordnung, II, Math. Zeit. 72 (1959), 25-46.

5. - Some applications of the theory of blocks of characters of finite groups, I, J. of Algebra 1 (1964), 152-167.

6. R. Brauer and H. F. Tuan, On simple groups of finite order, I, Bull. Amer. Math. Soc. 51 (1945), 756-766.

7. C. W. Curtis and I. Reiner, Representation theory of finite groups and associative algebras, Interscience, New York, London, 1962.

8. W. Feit, Characters of finite groups, W. A. Benjamin, New York, 1967.

9. D. Higman and C.C. Sims, A simple group of order 44,352,000, Math. Zeit. 105 (1968), 110-113.

10. B. Huppert, Endliche Gruppen, I, Springer-Verlag, Berlin, Heidelberg, New York, 1967.

11. Z. Janko, A characterization of the Mathieu simple groups, I, J. of Algebra 9 (1968), 1-19.

12. D. Parrott, On the Mathieu groups $M_{22}$ and $M_{11}$, Australian J. Math. (to appear) 13. J. G. Thompson, Nonsolvable finite groups all of whose local subgroups are solvable, Bull. Amer. Math. Soc. 74 (1968), 383-437.

14. S. K. Wong, On a new finite non-abelian simple group of Janko, Australian J. Math. (to appear)

15. W. J. Wong, Exceptional character theory and the theory of blocks, Math. Zeit. 91 (1966), 363-379.

Received January 8, 1969.

MONASH UNIVERSITY

ClaytoN 


\section{PACIFIC JOURNAL OF MATHEMATICS}

\section{EDITORS}

\author{
H. SAMELSON \\ Stanford University \\ Stanford, California 94305 \\ RICHARD PIERCE \\ University of Washington \\ Seattle, Washington 98105
}

J. DugundJI

Department of Mathematics University of Southern California Los Angeles, California 90007

BASIL GORDON*

University of California

Los Angeles, California 90024

\section{ASSOCIATE EDITORS}
E. F. BECKENBACH
B. H. NEUMANN
F. WOLE
K. YOSHIDA

\section{SUPPORTING INSTITUTIONS}

\author{
UNIVERSITY OF BRITISH COLUMBIA \\ CALIFORNIA INSTITUTE OF TECHNOLOGY \\ UNIVERSITY OF CALIFORNIA \\ MONTANA STATE UNIVERSITY \\ UNIVERSITY OF NEVADA \\ NEW MEXICO STATE UNIVERSITY \\ OREGON STATE UNIVERSITY \\ UNIVERSITY OF OREGON \\ OSAKA UNIVERSITY \\ UNIVERSITY OF SOUTHERN CALIFORNIA
}

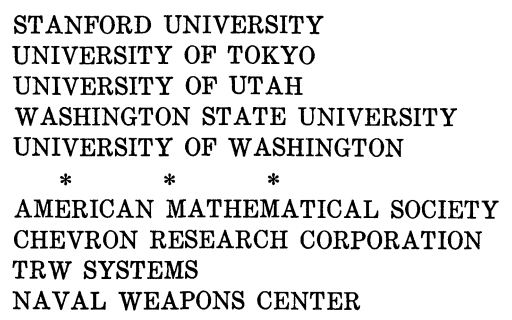

The Supporting Institutions listed above contribute to the cost of publication of this Journal, but they are not owners or publishers and have no responsibility for its content or policies.

Mathematical papers intended for publication in the Pacific Journal of Mathematics should be in typed form or offset-reproduced, (not dittoed), double spaced with large margins. Underline Greek letters in red, German in green, and script in blue. The first paragraph or two must be capable of being used separately as a synopsis of the entire paper. The editorial "we" must not be used in the synopsis, and items of the bibliography should not be cited there unless absolutely necessary, in which case they must be identified by author and Journal, rather than by item number. Manuscripts, in duplicate if possible, may be sent to any one of the four editors. Please classify according to the scheme of Math. Rev. 36, 1539-1546. All other communications to the editors should be addressed to the managing editor, Richard Arens, University of California, Los Angeles, California, 90024.

50 reprints are provided free for each article; additional copies may be obtained at cost in multiples of 50 .

The Pacific Journal of Mathematics is published monthly. Effective with Volume 16 the price per volume (3 numbers) is $\$ 8.00$; single issues, $\$ 3.00$. Special price for current issues to individual faculty members of supporting institutions and to individual members of the American Mathematical Society: $\$ 4.00$ per volume; single issues $\$ 1.50$. Back numbers are available.

Subscriptions, orders for back numbers, and changes of address should be sent to Pacific Journal of Mathematics, 103 Highland Boulevard, Berkeley, California, 94708.

PUBLISHED BY PACIFIC JOURNAL OF MATHEMATICS, A NON-PROFIT CORPORATION

Printed at Kokusai Bunken Insatsusha (International Academic Printing Co., Ltd.), 7-17, Fujimi 2-chome, Chiyoda-ku, Tokyo, Japan.

* Acting Managing Editor. 


\section{Pacific Journal of Mathematics}

\section{Vol. 32, No. $2 \quad$ February, 1970}

Harry P. Allen and Joseph Cooley Ferrar, Jordan algebras and exceptional subalgebras of the exceptional algebra $E_{6} \ldots \ldots \ldots \ldots \ldots \ldots \ldots 283$

David Wilmot Barnette and Branko Grünbaum, Preassigning the shape of a

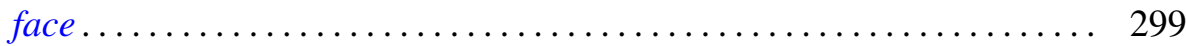

Robert Francis Craggs, Involutions of the 3-sphere which fix 2-spheres . . . . 307

David William Dean, Bor-Luh Lin and Ivan Singer, On k-shrinking and $k$-boundedly complete bases in Banach spaces ................ 323

Martin Engert, Finite dimensional translation invariant subspaces ....... 333

Kenneth Lewis Fields, On the global dimension of residue rings ......... 345

Howard Gorman, The Brandt condition and invertibility of modules ....... 351

Benjamin Rigler Halpern, A characterization of the circle and interval ..... 373

Albert Emerson Hurd, A uniqueness theorem for second order quasilinear

hyperbolic equations ............................... 415

James Frederick Hurley, Composition series in Chevalley algebras ...... 429

Meira Lavie, Disconjugacy of linear differential equations in the complex

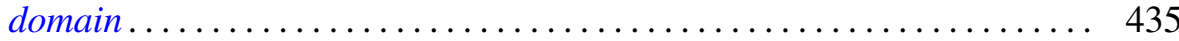

Jimmie Don Lawson, Lattices with no interval homomorphisms ......... 459

Roger McCann, A classification of center-foci ................. 467

Evelyn Rupard McMillan, On continuity conditions for functions . . . . . . . 479

Graciano de Oliveira, A conjecture and some problems on permanents .... 495

David L. Parrott and S. K. Wong, On the Higman-Sims simple group of order $44,352,000$.

Jerome L. Paul, Extending homeomorphisms ................. 517

Thomas Benny Rushing, Unknotting unions of cells .............. 521

Peter Russell, Forms of the affine line and its additive group.......... 527

Niel Shilkret, Non-Archimedean Gelfand theory ................. 541

Alfred Esperanza Tong, Diagonal submatrices of matrix maps.......... 551 